\title{
Apoptosis Imaging: Beyond Annexin V
}

\author{
Gang Niu ${ }^{1,2}$ and Xiaoyuan Chen ${ }^{1}$ \\ ${ }^{1}$ Laboratory of Molecular Imaging and Nanomedicine, National Institute of Biomedical Imaging and Bioengineering, National \\ Institutes of Health, Bethesda, Maryland; and ${ }^{2}$ Imaging Sciences Training Program, Radiology and Imaging Sciences, Clinical Center \\ and National Institute of Biomedical Imaging and Bioengineering, National Institutes of Health, Bethesda, Maryland
}

Induction of apoptosis is the primary mechanism through which most chemotherapies cause tumor cell death. Early assessment of tumor response is required to manage patients in terms of quality of life versus intensive chemotherapy. Although imaging with radiolabeled annexin $\mathrm{V}$ has been intensively investigated, it is still not sufficiently mature for clinical application. This article will summarize various alternative imaging techniques for visualization of phosphatidylserine externalization, activity of caspases, and mitochondrial membrane potential. Such imaging studies will promote the identification of novel molecular targets and the development of highly specific apoptosis-detecting imaging probes with potential clinical applications. It is highly possible that quantitative imaging of apoptosis will greatly improve clinical decision making in apoptosis-related diseases.

Key Words: apoptosis; molecular imaging; phosphatidylserine; caspases; optical imaging; PET

J Nucl Med 2010; 51:1659-1662

DOI: 10.2967/jnumed.110.078584

Apoptosis, or programmed cell death, plays a crucial role in many biologic processes and is involved in various diseases (1). The 2 best-studied mechanisms of apoptosis are the death receptor (extrinsic) and the mitochondrial (intrinsic) pathways, although there is significant cross-talk between the two (Fig. 1). Most chemotherapy agents cause tumor cell death by inducing apoptosis, whereas resistance to anticancer treatment is believed to involve mutations that lead to deregulated cellular proliferation and suppression of mechanisms that control apoptosis (1). Indeed, animal studies and early results in cancer patients imaged before and after either chemotherapy or irradiation with apoptotic imaging agents suggest that successful treatment is associated with a marked increase in tracer localization in the tumor (2).

Several potential steps in the apoptosis process could be imaged using various imaging modalities, including MRI, optical imaging, ultrasound, and radionuclide imaging. Several excellent review articles have stated that each imaging modality has certain advantages as well as limitations, and the choice of an imaging

Received May 19, 2010; revision accepted Jul. 16, 2010.

For correspondence or reprints contact either of the following:

Gang Niu, National Institutes of Health, 9 Memorial Dr., 9/1W111, Bethesda, MD 20892.

E-mail: niug@mail.nih.gov

Xiaoyuan Chen, National Institutes of Health, 31 Center Dr., 31/1C22,

Bethesda, MD 20892.

E-mail: shawn.chen@nih.gov

COPYRIGHT @ 2010 by the Society of Nuclear Medicine, Inc. modality, or combination of techniques, is determined by the specific biologic questions being asked $(3,4)$.

After caspase- 3 activation, there is a rapid redistribution and exposure of the anionic phospholipid phosphatidylserine on the cell surface. Phosphatidylserine exposure is a near-universal event in apoptosis. It occurs within a few hours of the apoptotic stimulus and presents an abundant target that is readily accessible on the extracellular face of the plasma membrane (5). Annexin $\mathrm{V}$-with its high affinity for apoptotic cells, lack of immunogenicity, and lack of in vivo toxicity - has been the most intensively studied imaging probe for in vivo apoptosis detection, especially with radionuclide labeling $(4,6)$. There are, however, several issues that limit further clinical use of radiolabeled annexin V probes. One concern is the suboptimal pharmacokinetics of radiolabeled annexin $V$ protein, especially the high background activity in the abdominal region. Another concern is the inability of annexin V probes to differentiate apoptosis from necrosis: phosphatidylserine is also exposed during necrosis subsequent to apoptosis, because of the disruption of plasma membrane integrity. In addition, optimal binding of annexin $\mathrm{V}$ to phosphatidylserine requires a micromolar concentration of $\mathrm{Ca}^{2+}$. Other imaging probes to visualize apoptosis in vivo that are being investigated will be briefly reviewed and commented on here, although the superiority of such probes to annexin $\mathrm{V}$ remains to be determined.

\section{IMAGING PHOSPHOLIPID REORGANIZATION}

\section{Imaging Apoptosis with Other Phosphatidylserine Binding Proteins}

Besides annexin $\mathrm{V}$, other proteins also show phosphatidylserine binding affinity. For example, the C2A domain of synaptotagmin I also binds to negatively charged phospholipids in membranes, including phosphatidylserine, in a calcium-dependent manner. C2A has been labeled with ${ }^{99 \mathrm{~m}} \mathrm{Tc}$ for SPECT of non-small cell lung cancer apoptosis induced by paclitaxel treatment. The results showed significantly increased tumor contrast after the induction of apoptosis by paclitaxel (7). ${ }^{99 \mathrm{~m}} \mathrm{Tc}-\mathrm{C} 2 \mathrm{~A}$-glutathione S-transferase has also been applied to a reperfused acute myocardial infarction rat model. Ex vivo and in vivo data indicate that both specific binding and passive leakage contribute to the accumulation of the radiotracer in the area at risk (8). A recent in vitro study suggested that the $\mathrm{C} 2 \mathrm{~A}$ derivative binds more specifically to apoptotic and necrotic cells than does annexin V (9).

\section{Imaging Apoptosis with Phosphatidylserine Binding Peptides and Small Molecules}

Compared with protein probes, peptides and small molecules have favorable characteristics, including rapid clearance from the circulation, efficient tissue penetration, and high target-to-nontarget 
FIGURE 1. Schematics of extrinsic and intrinsic apoptosis pathways. DR $=$ death receptor; FADD = Fas-associated death domain; FasL = Fas ligand; TNF = tumor necrosis factor; TRAIL = tumor necrosis factor-related apoptosis-inducing ligand.

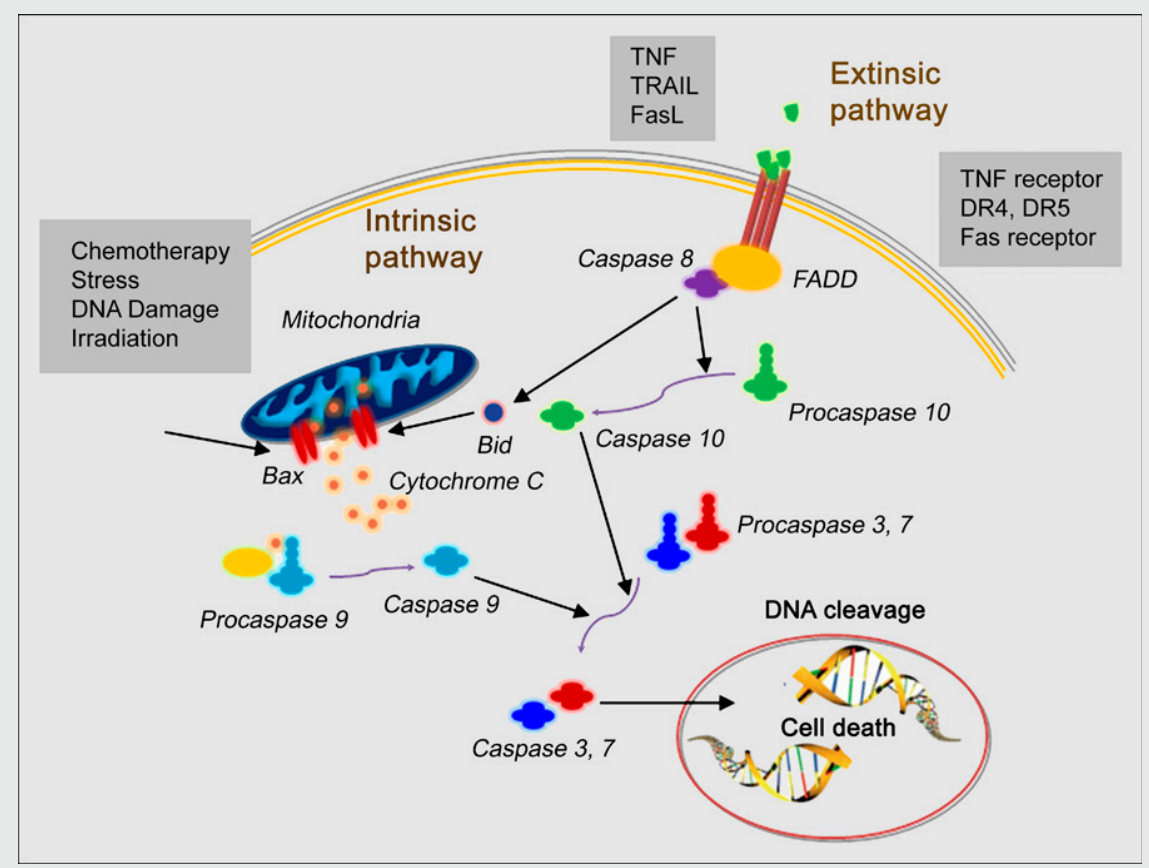

ratios. Consequently, several peptide sequences have been screened by various groups targeting to phosphatidylserine exposure in apoptotic cells using bacteriophage display technology. Optical imaging after the systemic administration of fluorescein-labeled CLSYYPSYC peptide to tumor-bearing nude mice (H460-cell xenograft model) treated with a single dose of camptothecin indicated peptide homing to the tumor (10). In another study, a library of linear 6-mer random peptides was screened in vitro against immobilized phosphatidylserine. Alignment of amino acid sequences of identified peptides with relevant proteins revealed a frequent homology with $\mathrm{Ca}^{2+}$ channels, reminiscent of the function of annexins (11).

ApoSense molecules are small nonpeptidic fluorescent compounds developed on the basis of Gla structure. In response to apoptosis, these molecules manifest selective membrane binding, transmembrane transport, and selective accumulation in the cytoplasm of apoptotic cells, while being excluded from viable cells $(12,13)$. Besides the fluorescence feature, some of these ApoSense molecules contain a fluorine atom, which facilitates radiolabeling with ${ }^{18} \mathrm{~F}$ isotope for PET. Recently, synthetic zinc(II)-dipicolylamine also showed potential to image apoptotic and necrotic cells in vivo (Fig. 2A) (14). However, further characterization of these apoptosis imaging probes is needed to fully explore the potential of these lowmolecular-weight alternatives to annexin V.

Apoptosis is a complicated and dynamic process that is still not fully understood. Even in vitro, the identification and characterization of apoptosis is not always straightforward, as necrosis can also produce DNA fragmentation, and with disrupted membranes annexin $\mathrm{V}$ can diffuse inside cells and stain intracellular phosphatidylserine. In addition, because not all apoptotic cells undergo DNA laddering or characteristic morphologic changes, there is no perfectly specific marker for apoptosis, even in vitro.

\section{IMAGING CASPASE ACTIVITY}

\section{Direct Imaging of Caspases}

Both intrinsic and extrinsic apoptosis pathways converge on a family of cysteine aspartate-specific proteases known as the cas- pases. By high-throughput screening and further structural optimization, several isatin ( ${ }^{1} \mathrm{H}$-indole-2,3-dione) sulfonamide analogs were identified to have nanomolar potency in inhibiting the executioner caspases, caspase-3 and caspase-7 (15). Small-animal PET using 1-[4-(2-18 F-fluoroethoxy)-benzyl]-5-(2-phenoxymethyl-pyrrolidine-1-sulfonyl)- $1 H$-indole-2,3-dione $\left({ }^{18} \mathrm{~F}\right.$-WC-II-89), one of the isatin sulfonamide analogs, revealed a high uptake of the radiotracer in the liver of a cycloheximide-treated rat, relative to the untreated control (Fig. 2C) (16). Because caspases are intracellular targets, the radiolabeled imaging probes are usually lipophilic or contain a cellpenetrating moiety to achieve target access, which dampens the enthusiasm for these probes (17).

Another problem for caspase imaging is that caspase inhibitors usually lack selectivity, as they are also effective inhibitors of various cathepsins. Recently, Bogyo's group identified irreversible inhibitors and active site probes of the caspases (activity-based probes) that showed both broad and narrow selectivity within this family of proteases. Further optimization identified sequences that showed lower legumain reactivity and a complete lack of reactivity toward the cathepsins (18). Optical imaging probes were developed by conjugating these activity-based probes with near-infrared fluorescent tags and a cell-permeable peptide sequence.

\section{Activatable Probes for Caspases}

The high background activity of caspase imaging with directly labeled probes can be overcome by activatable probes, which typically consist of 3 functional components. For example, a caspase-activatable probe, TcapQ(647), was synthesized to comprise a Tat-peptide-based permeation peptide sequence; an effector caspase recognition sequence, Asp-Glu-Val-Asp (DEVD); and a flanking optically activatable pair comprising a far-red quencher, QSY 21, and a fluorophore, Alexa Fluor 647. Under baseline conditions, high quenching efficiencies resulting in low background fluorescence were observed. On exposure to executioner caspases, TcapQ(647) was specifically cleaved, thereby releasing the fluorophore from the quencher and enabling imaging of apoptosis. In vivo experiments demonstrated the ability of TcapQ(647) to detect parasite-induced 


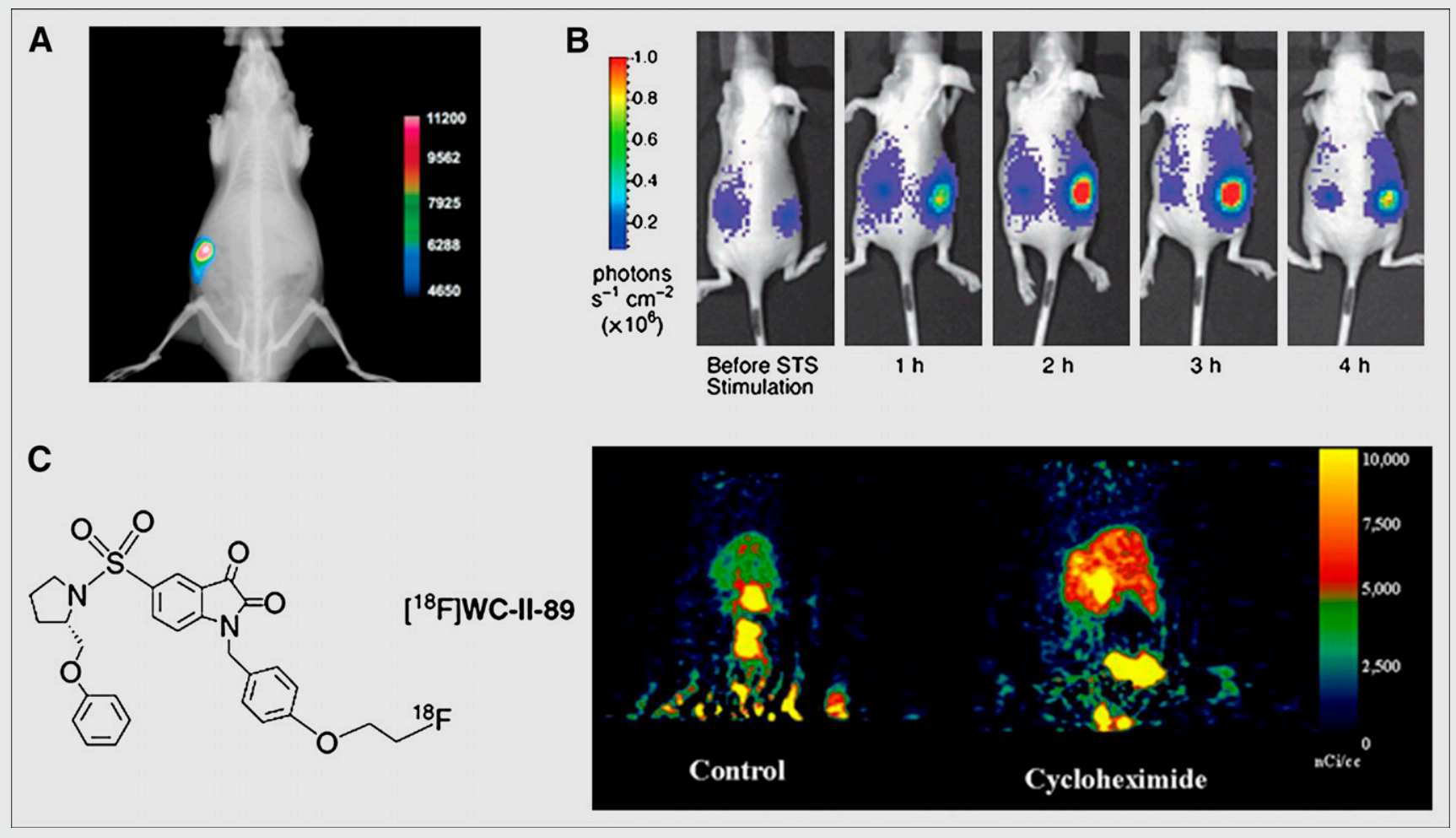

FIGURE 2. (A) Radiographic and fluorescence overlay image of rat prostate tumor model at $24 \mathrm{~h}$ after injection of near-infrared carbocyanine fluorophore-labeled zinc(II)-dipicolylamine. (Reprinted with permission of (14).) (B) In vivo bioluminescence imaging of mice carrying transiently transfected HeLa cells expressing cyclic Fluc (right side) and full-length Fluc (left side). Images were taken at the indicated times after intraperitoneal injection of staurosporine. (Reprinted with permission of (26).) (C) Molecular structure of ${ }^{18} \mathrm{~F}-\mathrm{WC}-\mathrm{II}-89$, an isatin sulfonamide analog, and whole-body small-animal PET images of ${ }^{18} \mathrm{~F}$-WC-II-89 distribution in control rat and cycloheximide-treated rat. (Reprinted with permission of (16).)

apoptosis in human colon xenograft and liver abscess mouse models (19). Cell-permeable polymeric nanoparticles have also been prepared for apoptosis imaging. The close spatial proximity of the near-infrared fluorochromes in polymeric nanoparticles resulted in an autoquenched state and strong near-infrared fluorescence signal emitted in apoptotic cells (20). Theranostic agents that combine therapeutic components and a caspase-activatable imaging moiety have also been reported (21).

Most activatable probes use the substrate DEVD to achieve caspase- 3 cleavage after being internalized into lysosomes. Legumain is a lysosomal cysteine protease that plays a pivotal role in the endosomal/lysosomal degradation system. Cathepsins also become activated at low $\mathrm{pHs}$ found in lysosomes. Thus, both cathepsins and legumain will digest DEVD and restore the optical signal even in nonapoptotic cells (18). The cross-reaction may result in nonspecific activation of the activatable probes and increase the background activity of in vivo imaging. Key directions of future development of activatable probes will be how to improve internalization and how to reduce noncaspase cleavage.

\section{Reporter Gene Imaging of Caspase Activity}

Bioluminescence imaging has been applied to image apoptosis with several seminal apoptosis-responsive reporter gene constructs (22-25). One reporter gene contains firefly luciferase gene flanked by ER (residues 281-599 of the modified mouse estrogen receptor sequence) with DEVD linker. A caspase-3-specific cleavage of the recombinant product results in the restoration of luciferase activity in cells undergoing apoptosis (22). Ray et al. made a fusion protein construct by combining 3 different reporter proteins, red fluorescent protein, firefly luciferase, and HSV1-sr39 truncated thymidine kinase, linked through a caspase- 3 recognizable polypeptide linker. On apoptosis induction with $8 \mu \mathrm{M}$ staurosporine, the fusion protein showed a significant increase in firefly luciferase and red fluorescent protein activity in 293T cells (24). A DEVD containing cyclic luciferase to detect caspase activation has also been reported (26). Two fragments of DnaE intein were fused to neighboring ends of firefly luciferase connected with a DEVD sequence. After translation into a single polypeptide in living cells, the amino and carboxy terminals of the luciferase were ligated by protein splicing, which resulted in a closed circular polypeptide chain. When the substrate sequence was digested by caspases, the luciferase was changed into an active form and restored its activity (Fig. 2B). Instead of modifying the reporter gene to respond to caspases, luciferase reporter gene substrate can also be modified to reflect caspase activity. One such example is a proluminescent, caspaseactivated DEVD-aminoluciferin reagent (Caspase-Glo 3/7; Promega) $(27,28)$.

\section{Imaging Mitochondrial Membrane Potential}

Another approach to detecting apoptotic cell death is targeting the collapse of mitochondrial membrane potential $\left(\Delta \psi_{\mathrm{m}}\right)$, a hallmark of the initiating phase of apoptosis (3). Phosphonium cations are sufficiently lipophilic to permeate the membrane lipid bilayer and accumulate in cells as a function of the transmembrane voltage 
gradient. Because of the high mitochondrial membrane potential, most of the phosphonium cations accumulate within mitochondria. A PET agent, ${ }^{18}$ F-fluorobenzyl triphenylphosphonium, demonstrated good uptake in H345 cells. Selective collapse of $\Delta \psi_{\mathrm{m}}$ caused a substantial decrease in cellular uptake of ${ }^{18} \mathrm{~F}$-fluorobenzyl triphenylphosphonium, compared with a control (29). In an orthotopic prostate tumor model, docetaxel caused a marked decrease $(52.4 \%)$ of ${ }^{18} \mathrm{~F}$-fluorobenzyl triphenylphosphonium tumor uptake within $48 \mathrm{~h}$, whereas ${ }^{18} \mathrm{~F}$-FDG was much less affected (12\%) (30). Compared with the transient nature of phosphatidylserine externalization, loss of $\Delta \psi_{\mathrm{m}}$ is an ongoing process not limited to a time window, which may allow time-independent detection of apoptosis. However, "negative" contrast due to decreased accumulation of the imaging probes in apoptotic cells and cellular efflux mediated by the multidrug-resistance proteins may limit its clinical application (3).

\section{CONCLUSION}

Conventional apoptosis detection methods, such as hematoxylin and eosin staining, flow cytometry, and deoxyuride-5' - triphosphate biotin nick end labeling assay, are complementary to noninvasive imaging techniques and provide critical standards to confirm the specificity of the apoptotic signal provided by either PET/SPECT or optical imaging methods. Besides annexin V probes, other radiolabeled proteins, peptides, or small molecules that recognize anionic phospholipid phosphatidylserine exposed on a cell surface remain questionable in their ability to truly reflect the intrinsically complex apoptotic process. Imaging of mitochondrial potential is often complicated by the presence of multidrug resistance proteins and, consequently, reduced cellular uptake and rapid efflux of the probes. Although the clinical use of optical imaging is limited, a highly sensitive activatable probe or reporter gene approach could provide a robust platform for preclinical drug screening and therapy monitoring.

Better understanding of numerous biochemical features of apoptosis may provide opportunities to develop new classes of imaging agents that outperform existing ones. Further improvement of apoptotic cell specificity and optimization of tracer pharmacokinetics to achieve increased target-to-nontarget ratios is needed to facilitate clinical translation and wide use of radiolabeled apoptosis probes. It is also essential to combine apoptosis imaging strategies with imaging of other critical biologic or pathologic pathways, such as metabolism, proliferation, hypoxia, and angiogenesis, to improve clinical decision making in apoptosis-related diseases and interventions.

\section{ACKNOWLEDGMENT}

This work is supported in part by an Imaging Sciences Training Fellowship jointly funded by the Radiology and Imaging Sciences Department of NIH Clinical Center and by the Intramural Research Program, NIBIB, NIH. We thank Dr. Henry S. Eden for proofreading the manuscript.

\section{REFERENCES}

1. Cotter TG. Apoptosis and cancer: the genesis of a research field. Nat Rev Cancer. 2009;9:501-507.

2. Blankenberg FG. Monitoring of treatment-induced apoptosis in oncology with PET and SPECT. Curr Pharm Des. 2008;14:2974-2982.

3. Tait JF. Imaging of apoptosis. J Nucl Med. 2008;49:1573-1576.

4. Blankenberg FG. In vivo detection of apoptosis. J Nucl Med. 2008;49(suppl 2):81S-95S
5. Boersma HH, Kietselaer BL, Stolk LM, et al. Past, present, and future of annexin A5: from protein discovery to clinical applications. J Nucl Med. 2005;46:20352050.

6. Blankenberg FG. Imaging the molecular signatures of apoptosis and injury with radiolabeled annexin V. Proc Am Thorac Soc. 2009;6:469-476.

7. Wang F, Fang W, Zhao M, et al. Imaging paclitaxel (chemotherapy)-induced tumor apoptosis with ${ }^{99 \mathrm{~m}} \mathrm{Tc} \mathrm{C} 2 \mathrm{~A}$, a domain of synaptotagmin I: a preliminary study. Nucl Med Biol. 2008;35:359-364.

8. Zhao M, Zhu X, Ji S, et al. ${ }^{99 \mathrm{~m} T c-l a b e l e d ~ C 2 A ~ d o m a i n ~ o f ~ s y n a p t o t a g m i n ~ I ~ a s ~ a ~}$ target-specific molecular probe for noninvasive imaging of acute myocardial infarction. J Nucl Med. 2006;47:1367-1374.

9. Alam IS, Neves AA, Witney TH, Boren J, Brindle KM. Comparison of the C2A domain of synaptotagmin-I and annexin- $\mathrm{V}$ as probes for detecting cell death. Bioconjug Chem. 2010;21:884-891.

10. Thapa N, Kim S, So IS, et al. Discovery of a phosphatidylserine-recognizing peptide and its utility in molecular imaging of tumour apoptosis. J Cell Mol Med. 2008;12:1649-1660.

11. Burtea C, Laurent S, Lancelot E, et al. Peptidic targeting of phosphatidylserine for the MRI detection of apoptosis in atherosclerotic plaques. Mol Pharm. 2009;6:1903-1919.

12. Damianovich M, Ziv I, Heyman SN, et al. ApoSense: a novel technology for functional molecular imaging of cell death in models of acute renal tubular necrosis. Eur J Nucl Med Mol Imaging. 2006;33:281-291.

13. Cohen A, Shirvan A, Levin G, Grimberg H, Reshef A, Ziv I. From the Gla domain to a novel small-molecule detector of apoptosis. Cell Res. 2009;19:625-637.

14. Smith BA, Akers WJ, Leevy WM, et al. Optical imaging of mammary and prostate tumors in living animals using a synthetic near infrared zinc(II)dipicolylamine probe for anionic cell surfaces. J Am Chem Soc. 2010;132: 67-69.

15. Podichetty AK, Wagner S, Schroer S, et al. Fluorinated isatin derivatives. Part 2. New N-substituted 5-pyrrolidinylsulfonyl isatins as potential tools for molecular imaging of caspases in apoptosis. J Med Chem. 2009;52:3484-3495.

16. Zhou D, Chu W, Rothfuss J, et al. Synthesis, radiolabeling, and in vivo evaluation of an ${ }^{18} \mathrm{~F}$-labeled isatin analog for imaging caspase-3 activation in apoptosis. Bioorg Med Chem Lett. 2006;16:5041-5046.

17. De Saint-Hubert M, Prinsen K, Mortelmans L, Verbruggen A, Mottaghy FM. Molecular imaging of cell death. Methods. 2009;48:178-187.

18. Edgington LE, Berger AB, Blum G, et al. Noninvasive optical imaging of apoptosis by caspase-targeted activity-based probes. Nat Med. 2009;15:967973.

19. Bullok KE, Maxwell D, Kesarwala AH, et al. Biochemical and in vivo characterization of a small, membrane-permeant, caspase-activatable far-red fluorescent peptide for imaging apoptosis. Biochemistry. 2007;46:4055-4065.

20. Kim K, Lee M, Park H, et al. Cell-permeable and biocompatible polymeric nanoparticles for apoptosis imaging. J Am Chem Soc. 2006;128:3490-3491.

21. Stefflova K, Chen J, Li H, Zheng G. Targeted photodynamic therapy agent with a built-in apoptosis sensor for in vivo near-infrared imaging of tumor apoptosis triggered by its photosensitization in situ. Mol Imaging. 2006;5:520-532.

22. Laxman B, Hall DE, Bhojani MS, et al. Noninvasive real-time imaging of apoptosis. Proc Natl Acad Sci USA. 2002;99:16551-16555.

23. Coppola JM, Ross BD, Rehemtulla A. Noninvasive imaging of apoptosis and its application in cancer therapeutics. Clin Cancer Res. 2008;14:2492-2501.

24. Ray P, De A, Patel M, Gambhir SS. Monitoring caspase-3 activation with a multimodality imaging sensor in living subjects. Clin Cancer Res. 2008;14: 5801-5809.

25. Kanno A, Umezawa Y, Ozawa T. Detection of apoptosis using cyclic luciferase in living mammals. Methods Mol Biol. 2009;574:105-114.

26. Kanno A, Yamanaka Y, Hirano H, Umezawa Y, Ozawa T. Cyclic luciferase for real-time sensing of caspase-3 activities in living mammals. Angew Chem Int Ed Engl. 2007;46:7595-7599.

27. Liu JJ, Wang W, Dicker DT, El-Deiry WS. Bioluminescent imaging of TRAILinduced apoptosis through detection of caspase activation following cleavage of DEVD-aminoluciferin. Cancer Biol Ther. 2005;4:885-892.

28. Hickson J, Ackler S, Klaubert D, et al. Noninvasive molecular imaging of apoptosis in vivo using a modified firefly luciferase substrate, Z-DEVDaminoluciferin. Cell Death Differ. 2010;17:1003-1010.

29. Madar I, Ravert H, Nelkin B, et al. Characterization of membrane potentialdependent uptake of the novel PET tracer ${ }^{18} \mathrm{~F}$-fluorobenzyl triphenylphosphonium cation. Eur J Nucl Med Mol Imaging. 2007;34:2057-2065.

30. Madar I, Huang Y, Ravert H, et al. Detection and quantification of the evolution dynamics of apoptosis using the PET voltage sensor ${ }^{18} \mathrm{~F}$-fluorobenzyl triphenyl phosphonium. J Nucl Med. 2009;50:774-780. 